\title{
lean canvas aplicado a una idea de negocios de una bebida energizante en el municipio de pasto - nariño
}

\section{NOMBRE DE AUTORES}

\author{
Alicia Cristina Silva Calpa ${ }^{1}$ \\ Juan Carlos Díaz Dávila² \\ Luz Elena Montoya Rendón ${ }^{3}$
}

\section{RESUMEN}

El presente documento tiene como objetivo presentar el diseño del modelo de negocio para bebidas energizantes naturales saludables a base de Borojó en el mercado del municipio de Pasto - Nariño, a partir del antecedente fundamental de la connotación cultural de dicha fruta como un potencializador orgánico de las energías y de su fama de producto afrodisíaco.

Dado lo anterior y con el objeto de diseñar un negocio para la explotación de ésta potencialidad, se analiza la literatura que refleja el estado del arte del modelado de negocios, tras lo cual se encuentra que actualmente tiene relevancia el diseño de negocios con base en la metodología canvas, que desde su creación y hasta el presente ha evolucionado sustancialmente llegando al modelado Lean de Ash Mayra, que se usa en el presente trabajo para alcanzar el objetivo planteado, en la medida que este modelo, bajo los lineamientos metodológicos de canvas, es el más acorde para el caso de empresas en creación.

Palabras claves: modelo de negocios; magro; canvas; producto mínimo viable (PMV); iteración; comprador temprano.

${ }^{1}$ Administradora de empresas, Especialista en Pedagogía para el Desarrollo del Aprendizaje Autónomo, Magister en Sistemas de Calidad y Productividad, PhD en Administración. Docente Ocasional UNAD - CCAV Pasto, grupo de Investigación Sindamanoy, correo electrónico alicia.silva@unad.edu.co, C.C. 59823146 de Pasto, Código ORCID orcid.org/0000-0002-9329-0777

2 Magister en Administración de Empresas, Esp. en Gerencia de Negocios Internacionales y profesional en Administración de Empresas. Dirección, gerencia, consultoría, asesoría, docencia y capacitación en áreas económico administrativas en general. Experiencia de varios años en empresas y entidades de los sectores público, privado y del social. e-Mail: juank2607@gmail.com

3 Administradora de Empresas, Maestría en Administración de Empresas con Énfasis en Sistemas Integrados de Gestión. Investigador asociado del Grupo de Investigación I lama. Docente de la Universidad Nacional Abierta y a Distancia - UNAD, Colombia. luz.montoya@unad.edu.co 


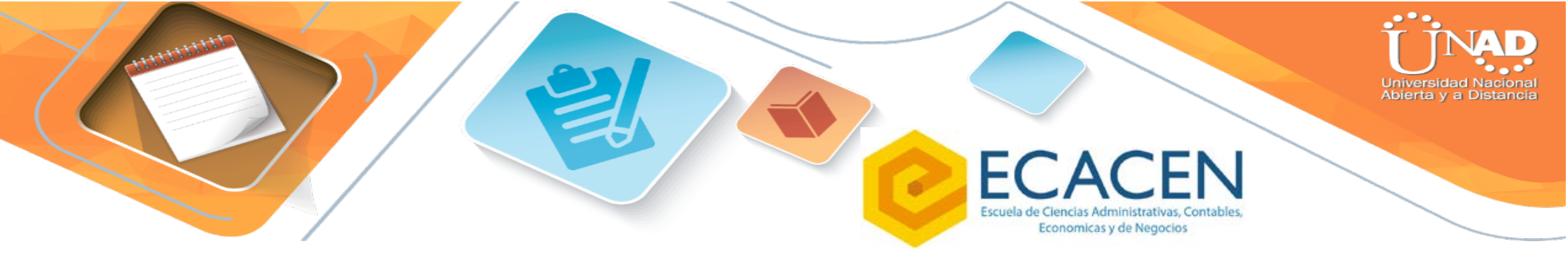

Abstract

This article aims to present the design of the business model for healthy natural energy drinks based on Borojó in the market of the municipality of Pasto - Nariño, starting from the fundamental antecedent of the cultural connotation of this fruit as an organic potentiator of the energies and its fame of aphrodisiac product.

Given the above and in order to design a business to exploit this potential, we analyze the literature that reflects the state of the art of business modeling, after which it is found that currently has relevance the design of businesses based on the canvas methodology, which since its creation and to the present has evolved substantially arriving at the Lean modeling of Ash Mayra, which is used in the present work to reach the objective set, insofar as this model, under the methodological guidelines of canvas, is the most appropriate for the case of start-ups.

Keywords: business model; read; canvas; minimum viable product (PMV); iteration; early adopter.

\section{NTRODUCCI ÓN}

El negocio de las bebidas no alcohólicas, que hace parte del subsector de alimentos y bebidas del segundo sector de la economía colombiana, ha tenido una gran transformación en los últimos años, puesto que los consumidores hoy por hoy son conscientes de la importancia de consumir bebidas saludables. Hace 10 años, el consumo de gaseosas representaba un poco más del $80 \%$ del consumo nacional del sector de las bebidas; sin embargo hoy en día, mientras que éstas luchan por no seguir perdiendo terreno, los jugos, las aguas y otros refrescos siguen ganando participación en el mercado. (SENA, 2008)

El mercado del Sector de Bebidas en su gran mayoría está representado por las empresas grandes y medianas, empresas líderes que se caracterizan por su capacidad para invertir, desarrollar gente, procesos y plantear propuestas nuevas para el mercado. Mientras que hay un grupo grande de microempresarios que están incursionando con aguas y jugos que no tienen el desarrollo tecnológico, productivo y ocupacional que haga peso dentro de un sector de crecimiento. (SENA, 2008)

Por otro lado, el ámbito de los negocios de hoy en día obliga a los empresarios y emprendedores a ser imaginativos y a salir de los esquemas, incluso a romper paradigmas de consumo que han prevalecido por mucho tiempo. Los retos del mercado hacen que sea necesaria y determinante una nueva manera de concebir los negocios, para lo cual se requieren nuevos métodos de planeamiento que tengan como eje central al consumidor, pues es éste quien al final define el éxito o el fracaso de los mismos. En las condiciones de competitividad actual, se hace indispensable contar con modelos que posibiliten detectar con anticipación y con la mayor precisión posible las diferenciaciones que le permitirán a un bien o servicio prosperar en los mercados, y dado que estás pueden ser de carácter distinto y en relación con el precio, el canal de comercialización, la presentación o la forma y las características del producto, dichos modelos deben ser lo más precisos posibles y ajustados a los requerimientos particulares de dichos mercados. 


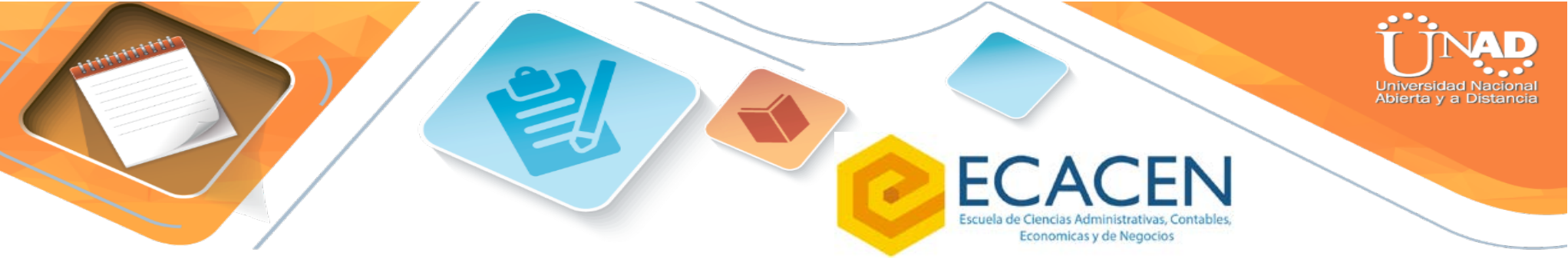

En cuanto a la metodología, se trata de una investigación cuantitativa dado que se suministra información para la evaluación de datos primarios y secundarios a fin de proveer cursos alternativos de acción. Este tipo de investigación se sub clasifica a su vez en investigación descriptiva ya que en ella se pueden detallar en forma gráfica los fenómenos y características del análisis de mercado, se determina la asociación de variables y con fundamento en ello se hacen los presupuestos y predicciones necesarios. En el marco de esta metodología, la presente investigación desarrolla el proceso de modelado de negocios de Ash Maurya atendiendo estrictamente a los pasos que para ello se describen en su libro "Running Lean - Iterando de un plan A a uno que funcione" (Maurya, 2012).

Para el correcto desarrollo del proceso de identificación del modelo de negocios y con base en la idea inicial que es la de ofrecer el producto al mercado objetivo conformado por hombres y mujeres económicamente activos, ocupados formalmente, comprendidos entre 14 y 65 años, del casco urbano del municipio de San Juan de Pasto, departamento de Nariño - Colombia en el año 2016, se siguen los pasos descritos en el siguiente esquema (Tabla. 3) que parte del análisis del entorno y continúa con la definición del modelo de negocio.

Figura 1. Proceso de desarrollo de la investigación

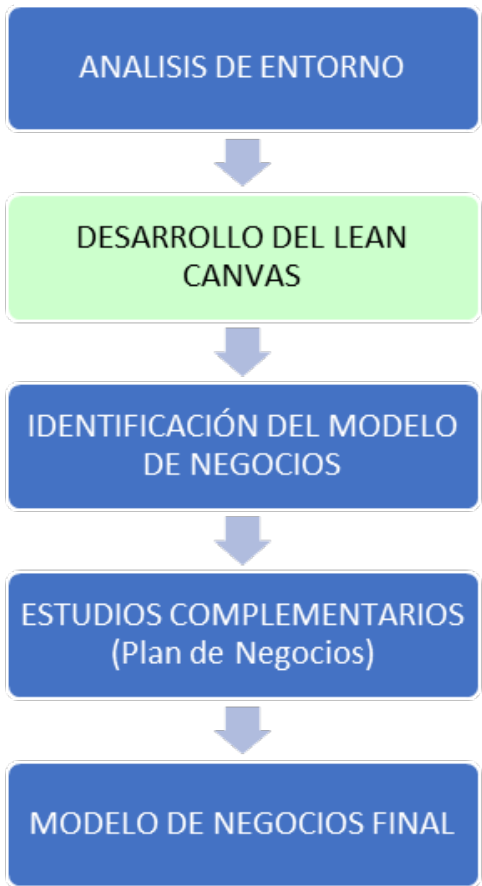

Fuente: El Autor 


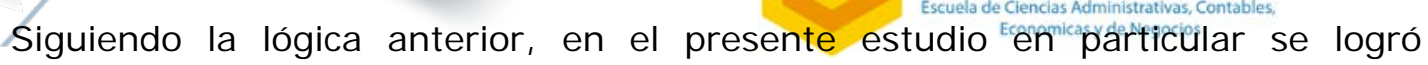
determinar como atributos fundamentales para el cliente los siguientes: producto natural, precio cómodo y producto asequible

Reduciendo al mínimo los recursos necesarios para la experimentación, se diseña un MVP (Fig. 3) que consiste en un frasco pet de $250 \mathrm{ml}$. Que tiene la siguiente presentación: El contenido del frasco es el jugo natural de Borojó con sabor a guanábana que a juicio del autor del presente estudio, es el que más neutraliza el sabor acido concentrado del borojó y le da a la bebida final el exquisito sabor que la caracteriza.

Las hipótesis de valor que caracterizan el producto se verifican mediante la aplicación a los tenderos de la siguiente ficha - encuesta que deben completar los compradores - consumidores (Fig. 4):

Dados los resultados de la iteración PRODUCTO/MERCADO, se puede concluir que estos son muy positivos para el MVP, y por tanto, habría que efectuar la iteración en el tamaño y la adecuación relacionado con esto en el eslogan.

Todos los hallazgos de las iteraciones se deben consignar en el canvas resultante, y estos a su vez corresponden a los ajustes que se deben realizar a los prototipos que se verifican en el mercado, dando lugar después de ello a nuevas posibles hipótesis que se plasman en nuevos canvas y se someten a nuevas iteraciones. 


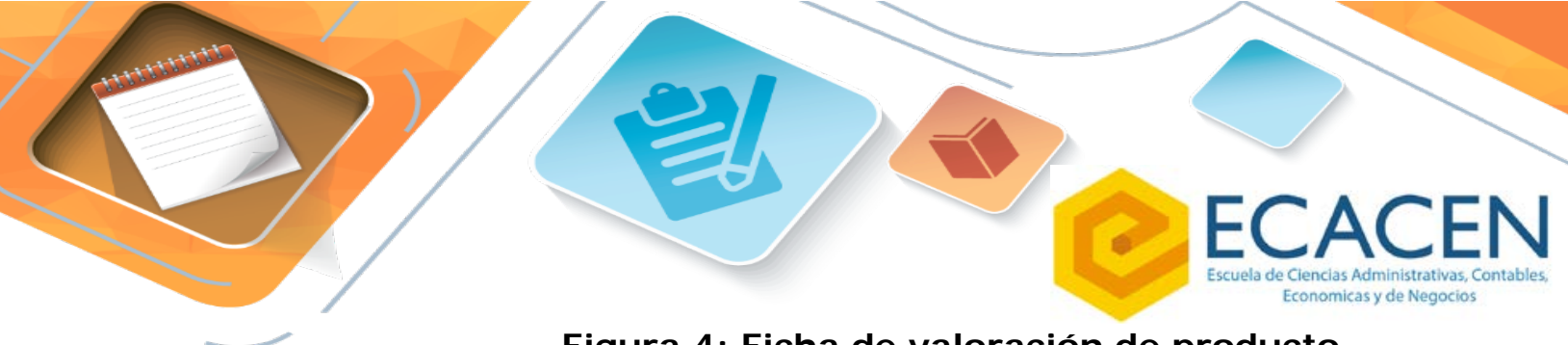

Figura 4: Ficha de valoración de producto

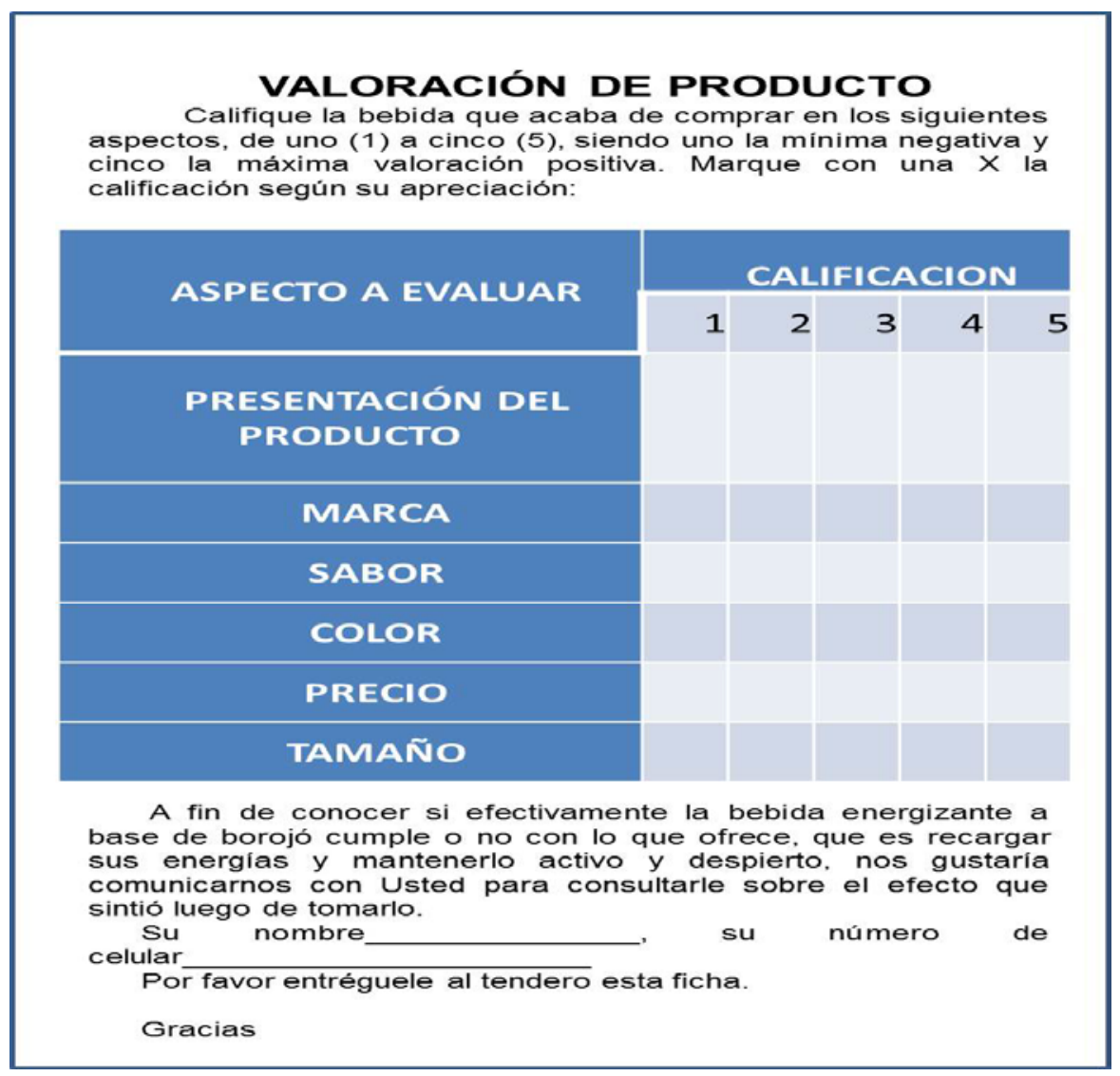

Fuente: El Autor

Los resultados obtenidos en los estudios de mercado, técnico, administrativo organizacional y financiero se incluyen como modificaciones al canvas, dando como resultado el canvas de modelo de negocio para una bebida energizante a base de borojó en el municipio de Pasto, cuyo nombre será "Borojizante" y que tendrá un precio asequibles de $\$ 1.000 .00$, cuyos consumidores adoptantes serán los estudiantes consumidores habituales de bebidas energizantes, para quienes el producto proveerá energía saludable y potencia que les ayudará a mantenerse despiertos y activos sin afectar su salud. El canal para acceder a los consumidores del producto serán las tiendas y restaurantes universitarios de fácil acceso a los estudiantes en los cuales se realizarán actividades de impulso en venta.

Se presenta a continuación (Fig. 5) el canvas resultante para una bebida energizante a desarrollarse en el municipio de Pasto, aclarando que las variables que no se resaltan en color azul han sido suficientemente expuestas en los anteriores canvas. Este diseño es susceptible de ajustes o iteraciones posteriores, basados en nuevos hallazgos y aprendizajes en las etapas de implementación y desarrollo. 


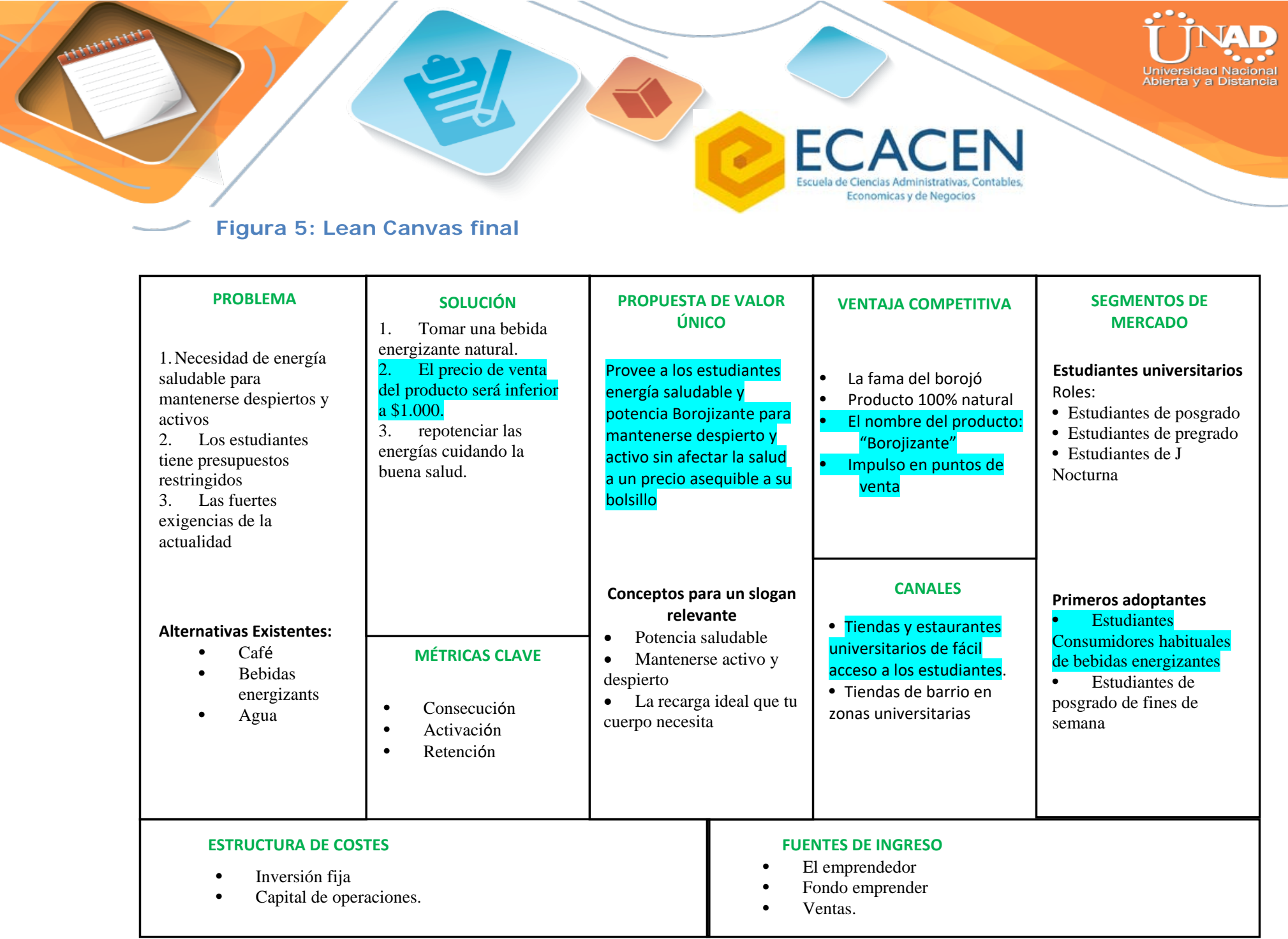

Fuente: El Autor 


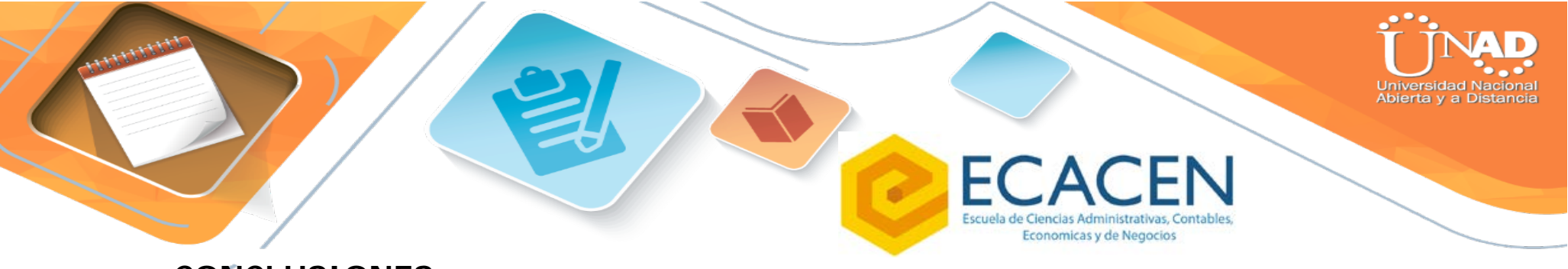

\section{CONCLUSIONES}

La construcción exitosa de un modelo de negocio se basa principalmente en la validación de las hipótesis que se identifiquen alrededor de la idea y en las sucesivas iteraciones, que permiten los ajustes necesarios al producto mínimo viable PMV, hasta llevarlo a través de la real puesta a prueba en su nicho de mercado básico (early adopter), a convertirse en un proyecto empresarial viable.

Resulta evidente que para garantizar en mayor medida el éxito en los emprendimientos empresariales de las micro y pequeñas empresas especialmente, se hace necesario contar, no solo con diseños de negocio apropiados, para lo cual el método Lean Canvas es adecuado, sino que además, estos sean complementados y soportados por los correspondientes análisis de mercado, técnico, administrativo y financiero que le permitan al emprendedor tener mayor claridad sobre todo el proceso de montaje de su idea de negocio y de su desarrollo en la etapa inicial.

\section{BI BLI OGRAFí A.}

Cohen, J. (2010). A smartbear. Obtenido de https://blog.asmartbear.com/notcompetitive-advantage. html

Gomez, D. (2013). Santiago de Cali.

Icontec. (2009). Norma técnica colombiana. NTC 3837. Bogotá: Icontec.

Maurya, A. (2012). Lean Canvas. Deutso.

Osterwalder, A. (2010). Business model generation. New Jersey.

Pereira, J. E. (19 de Febrero de 2014). Mercadeo.com. Recuperado el 22 de Junio de 2015, de http://www.mercadeo.com/blog/2014/02/nuevos-modelos-denegocios/

SENA. (2008). Caracterización Ocupación del Sector de Bebidas Caracterización Ocupación del Sector de Bebidas. Bogotá DC: SENA.

Torres C, G., \& Quito R, L. (2007). Estudio de prefactibilidad técnico económica de una planta para elaborar una bebida a base de noni y borojó. Quito.

Weinberger. (2009). Herramientas para evaluar la viabilidad de un negocio. Lima, Perú. 